\title{
Variable $360^{\circ}$ Vector-Sum Phase Shifter With Coarse and Fine Vector Scaling
}

\author{
Mohammad-Mahdi Mohsenpour, Member, IEEE, and Carlos E. Saavedra, Senior Member, IEEE
}

\begin{abstract}
A CMOS vector-sum phase shifter covering the full $360^{\circ}$ range is presented in this paper. Broadband operational transconductance amplifiers with variable transconductance provide coarse scaling of the quadrature vector amplitudes. Fine scaling of the amplitudes is accomplished using a passive resistive network. Expressions are derived to predict the maximum bit resolution of the phase shifter from the scaling factor of the coarse and fine vector-scaling stages. The phase shifter was designed and fabricated using the standard 130-nm CMOS process and was tested on-wafer over the frequency range of 4.9-5.9 GHz. The phase shifter delivers root mean square (rms) phase and amplitude errors of $1.25^{\circ}$ and $0.7 \mathrm{~dB}$, respectively, at the midband frequency of $5.4 \mathrm{GHz}$. The input and output return losses are both below $17 \mathrm{~dB}$ over the band, and the insertion loss is better than $4 \mathrm{~dB}$ over the band. The circuit uses an area of $0.303 \mathrm{~mm}^{2}$ excluding bonding pads and draws $28 \mathrm{~mW}$ from a $1.2 \mathrm{~V}$ supply.
\end{abstract}

Index Terms-Active phase shifter, active summing junction, clock and data recovery, CMOS, IEEE 802.11n, LTE, monolithic microwave integrated circuit (MMIC), operational transconductance amplifiers (OTAs), phased array, quadrature generation, radar, RFIC, root mean square (rms) error, WiMAX.

\section{INTRODUCTION}

$\mathbf{T}$ HERE is continued interest in finding new methods to improve the resolution and accuracy of monolithic microwave integrated circuit (MMIC) phase shifters. That interest is motivated by the critical role that phase shifters have in multiple-input multiple output radio links and phased arrays. Design advances over the past decade have led to significant improvements in the fractional bandwidth of the phase shifters and a reduction in the footprint area of the chips. MMIC phase shifters covering the full $360^{\circ}$ using different techniques, such as delay lines [1], [2], signal reflection [3], highpass/low-pass networks [4], all-pass networks [5], and vector summation [6]-[12].

In vector-sum phase shifters, there often appear unreachable phase angles (phase gaps) at the quadrant edges that limit the phase-step (bit) resolution of digital phase shifters. The objective of this paper is to explore the issue of phase gaps in vector-sum phase shifters and to propose a solution to mitigate them. The general approach taken here is to use a twostep vector-scaling procedure. First, a coarse scaling is carried

Manuscript received April 6, 2015; revised August 18, 2015, February 9, 2016, and April 25, 2016; accepted May 21, 2016. Date of publication June 14, 2016; date of current version July 7, 2016. This work was supported by the Natural Science and Engineering Research Council of Canada.

The authors are with the Department of Electrical and Computer Engineering, Queen's University, Kingston, ON K7L 3N6, Canada (e-mail: m.mohsenpour@queensu.ca; saavedra@queensu.ca).

Color versions of one or more of the figures in this paper are available online at http://ieeexplore.ieee.org.

Digital Object Identifier 10.1109/TMTT.2016.2574843 out in the current domain using operational transconductance amplifiers (OTAs), and subsequently, fine scaling is done on the signal vector in the voltage domain using a resistive network before the signal vectors are added together. A prototype phase shifter was designed for the 5.4-GHz band and was fabricated using the 130-nm CMOS technology. Experimental test results are presented, which validate the concept.

\section{Phase Shifter Concept}

Fig. 1 shows the block diagram of the proposed MMIC phase shifter, where the shaded area shows the on-chip circuitry. The phasor diagrams above the shaded area illustrate how a representative input signal is modified as it propagates through the phase shifter. The external $180^{\circ}$ power splitter converts the RF input voltage signal, $v_{\mathrm{RF}}$, into a differential waveform. A quadrature generator then produces four equalamplitude orthogonal voltage signal vectors, $\pm v_{I}$ and $\pm v_{Q}$, for the $I$ and $Q$ paths, respectively. A pair of identical OTAs are used to scale the magnitude of the four voltage signal vectors and to convert them into current signals: $\pm i_{I, Q}= \pm G_{m} v_{I, Q}$, where $G_{m}$ is the transconductance gain of the OTAs, which is tuned through the analog control voltages $V_{\text {tune, } I}$ and $V_{\text {tune, }} Q$.

Two single-pole double-throw (SPDT) switches are used to select which $I$-path vector (the $0^{\circ}$ or the $180^{\circ}$ ) and which $Q$-path vector (the $90^{\circ}$ or the $270^{\circ}$ ) will be summed together at the output to produce the desired output phase angle. The SPDT switches are controlled using two digital bits, $a_{2} b_{2}$.

While the minimum gain, $G_{\min }$, of the OTAs can ostensibly be reduced to zero, the problem with doing so is that the phase response of the OTAs at zero gain can be quite different than at moderate to high gain levels, thereby compromising the root mean square (rms) phase and amplitude error performance of the circuit. As a result, there is a practical limit to how small $G_{\min }$ should be and that value can be found by observing the phase response of the OTA as a function of its gain.

Suppose now that $G_{\min }$ has been established and that the highest gain setting of the OTAs is denoted by $G_{\max }$, then the smallest output angle that the phase shifter would produce in quadrant $I$ is

$$
\theta_{\min }=\tan ^{-1}\left(\frac{G_{\min , Q}}{G_{\max , I}}\right) \mathrm{rad}
$$

as shown in Fig. 2(a). It is straightforward to see that there will be a range of phase angles that the phase shifter cannot produce between quadrants I and IV and at every other quadrant boundary, as shown in Fig. 2(b). These unreachable output phase angle regions are the so-called phase gaps. The size of the gaps is $\theta_{\text {gap }}=2 \theta_{\min }$ and they place a limit on the bit 


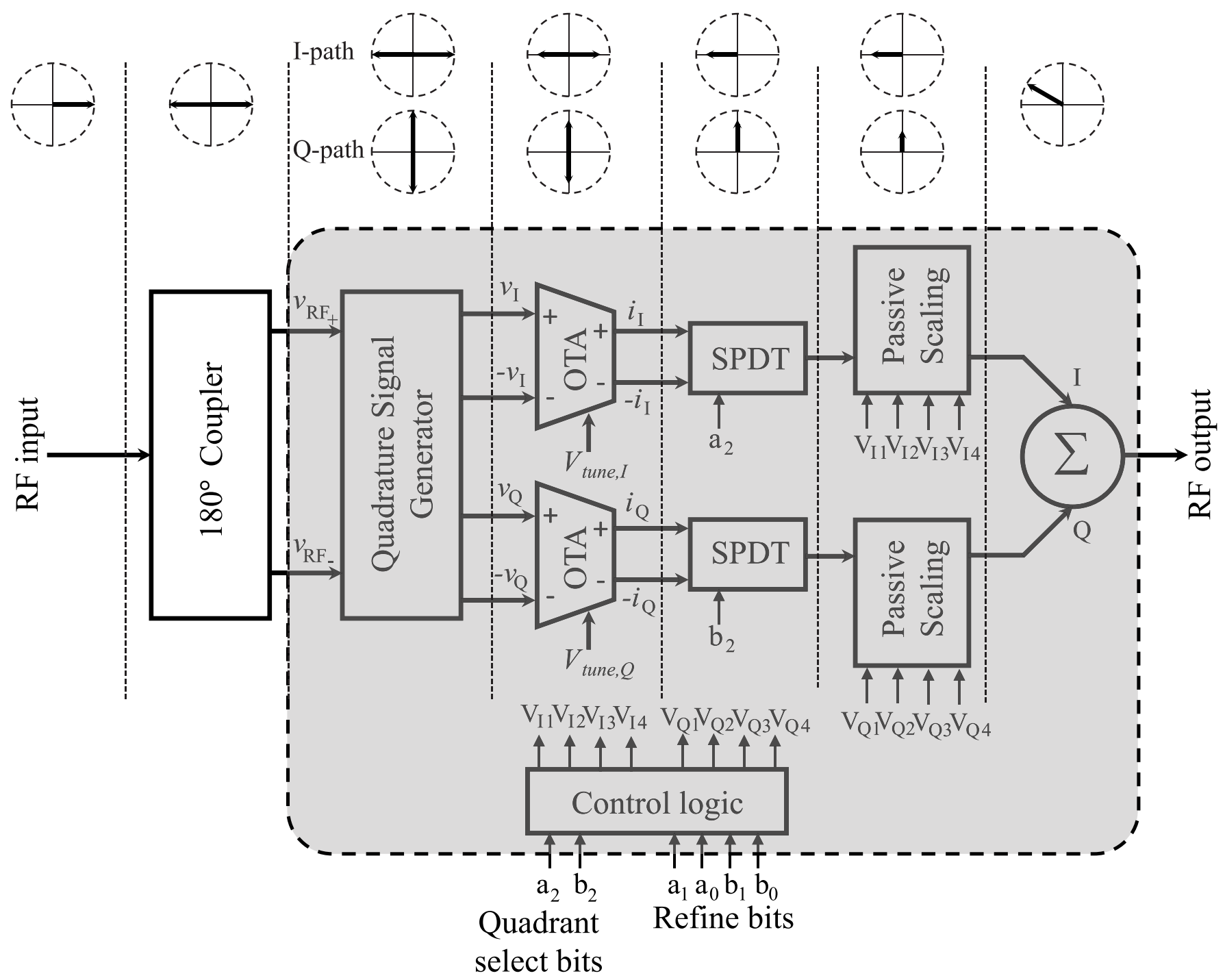

Fig. 1. Block diagram of the $360^{\circ}$ vector-sum phase shifter proposed in this paper. Components outside the shaded area are off-chip.
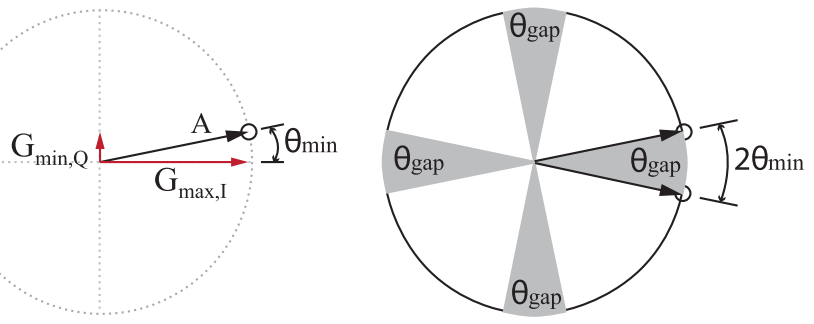

(a)

(b)
Fig. 2. (a) Minimum output phase angle, $\theta_{\min }$, that can be produced in quadrant I. (b) Phase gaps equal to $2 \theta_{\mathrm{min}}$ occur at the quadrant boundaries.

resolution of digital vector-sum phase shifters, since the circuit cannot have a phase step smaller than $\theta_{\text {gap }}$. The relationship between the phase gap and the phase-step resolution of the phase shifter is $\theta_{\text {gap }}<2 \pi / 2^{n}$, from which the maximum bit resolution is $\lfloor n\rfloor=\log _{2}\left(2 \pi / \theta_{\text {gap }}\right)$, where $\lfloor\cdot\rfloor$ denotes the floor function. With the aid of (1), the expression for the maximum bit resolution, $n$, of the phase shifter as a function of the amplifier gain tuning range is

$$
\lfloor n\rfloor=\log _{2}\left[\frac{\pi}{\tan ^{-1}\left(\frac{G_{\min , Q}}{G_{\max , I}}\right)}\right] .
$$

To reduce the size of $\theta_{\text {gap }}$ and thereby increase the bit resolution of the phase shifter, the proposed system in Fig. 1 employs the second vector-scaling step after the SPDT switches. This second scaling step is done with a resistive passive network. The final $I$ and $Q$ vectors are added using a summing junction to produce the desired phase-shifted signal.

\section{RFIC DESIGN}

This section provides design details of the phase shifter's building blocks in sequence from left to right. All circuit components were designed for a center frequency of $5.4 \mathrm{GHz}$. 


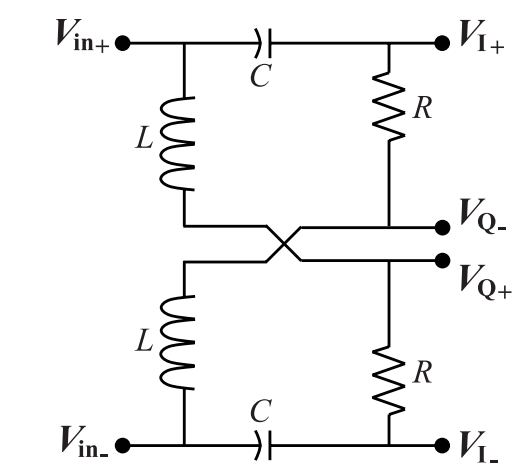

\begin{tabular}{|l|c|c|c|}
\hline Part & $L$ & $C$ & $R$ \\
\hline Value & $1.42 \mathrm{nH}$ & $0.6 \mathrm{pF}$ & $100 \Omega$ \\
\hline
\end{tabular}

Fig. 3. Schematic of the all-pass quadrature signal generator.

\section{A. Quadrature Signal Generator}

The circuit shown in Fig. 3 is used to generate differential quadrature basis vectors for the $I$ and $Q$ signal paths. It is an all-pass network that yields signals with tight amplitude and phase balance over wide bandwidths with a low return loss at the input port [6]. Using the component values shown in Fig. 3, the simulation results predict phase and amplitude imbalances less than $1^{\circ}$ and $0.35 \mathrm{~dB}$, respectively, and an input return loss below $16 \mathrm{~dB}$ for the quadrature generator over a $1-\mathrm{GHz}$ band centered at $5.4 \mathrm{GHz}$.

\section{B. OTA Vector-Scaling Stage (Coarse Scaling)}

The OTAs convert the incident voltage signals into currents. These signal currents are then scaled by varying the transconductance, $G_{m}$, of the OTAs. The OTA schematic is shown in Fig. 4 and is a variant of the circuit reported in [7] and [13]-[15]. Thus, only a basic description of the OTA is given here followed by the information relevant to the phase shifter design. The input signal feeds to $M_{1} / M_{2}$ and $M_{5} / M_{6}$ through $C_{1} / C_{2}$ and $C_{5} / C_{6}$, respectively. Transistors $M_{3}$ and $M_{4}$ are cross-coupled to provide feedforward regulation to the OTA for broadband operation and increased linearity. Tuning of the $G_{m}$ is done by changing the gate voltage of $M_{3} / M_{4}$ at the node labeled $V_{\text {tune }}$ in Fig. 4. Triple well nMOS devices are used here to provide source-body isolation for all OTA's devices and better isolation from substrate. Capacitors $C_{3}$ and $C_{4}$ are for dc blocking, and resistors $R_{1}-R_{6}$ have a large value and are used for dc biasing.

A key design goal for the OTA for the application at hand is for its gain, $G_{m}$, versus $V_{\text {tune }}$ relationship to have a linear response, so that the vector scaling also exhibits a linear dependence on the tuning voltage. A simulation of $G_{m}$ versus control voltage, $V_{\text {tune }}$, at a frequency of $5.4 \mathrm{GHz}$ is shown in Fig. 5. The magnitude of $G_{m}$ varies linearly from 5.5 to $32 \mathrm{mS}$ as $V_{\text {tune }}$ is swept from 0.45 to $0.85 \mathrm{~V}$. Therefore, $\theta_{\text {gap }}=2 \tan ^{-1}(5.5 / 32)=0.34 \mathrm{rad}=19.5^{\circ}$ and (2) predicts that the highest resolution that the phase shifter could produce is $4 \mathrm{~b}$ which corresponds to a phase step of $22.5^{\circ}$. To improve

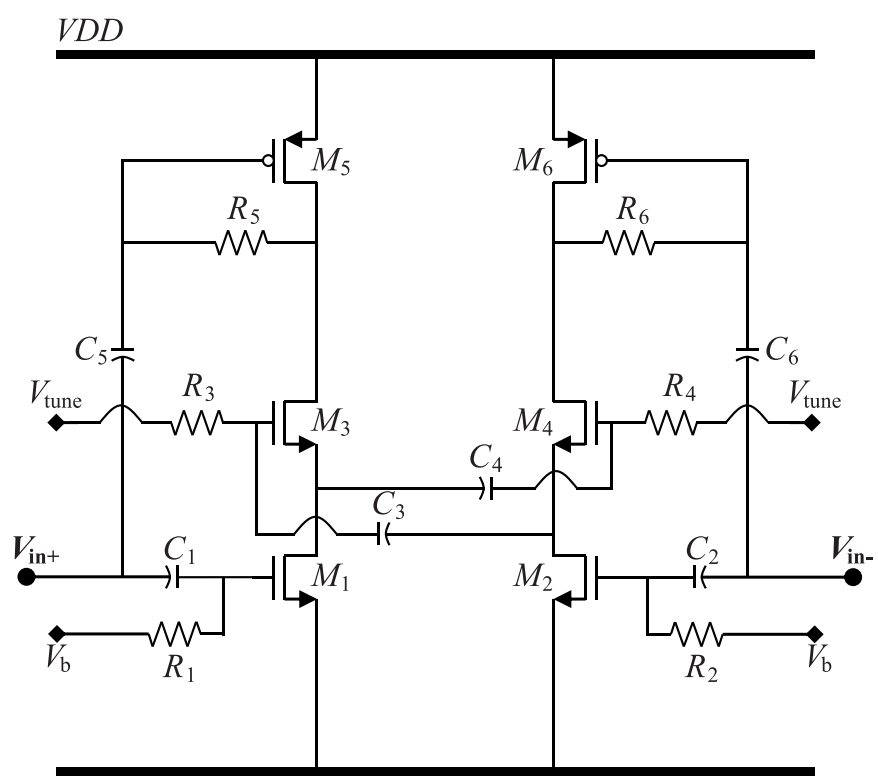

$\overline{V S S}$

\begin{tabular}{|l|c|c|c|}
\hline Part & $\left(\frac{W}{L}\right)_{1,2}$ & $\left(\frac{W}{L}\right)_{3,4}$ & $\left(\frac{W}{L}\right)_{5,6}$ \\
\hline Value & $\frac{90 \mu \mathrm{m}}{0.12 \mu \mathrm{m}}$ & $\frac{39 \mu \mathrm{m}}{0.12 \mu \mathrm{m}}$ & $\frac{90 \mu \mathrm{m}}{0.12 \mu \mathrm{m}}$ \\
\hline \hline Part & $R_{1-6}$ & $C_{1-2,5-6}$ & $C_{3,4}$ \\
\hline Value & $13.5 \mathrm{k} \Omega$ & $2.4 \mathrm{pF}$ & $1.68 \mathrm{pF}$ \\
\hline
\end{tabular}

Fig. 4. Schematic of the tunable feedforward-regulated OTA for coarse vector scaling.

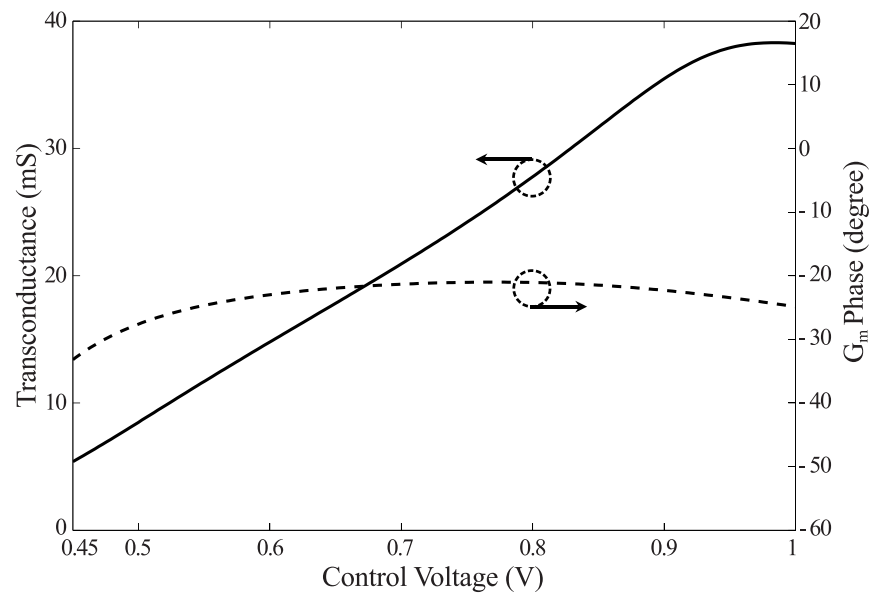

Fig. 5. Simulation results for amplitude and phase variations of the OTA versus control voltage.

the resolution of the phase shifter, the second scaling circuit is used to reduce the value of $G_{m}$. That circuit is described further below after the SPDT switches.

\section{SPDT Switches}

Two SPDT switches, connected to the OTAs' outputs, choose between the four quadrants. To keep the insertion loss of the switches below $1 \mathrm{~dB}$, two series nMOS transistor, $M_{7}$ and $M_{8}$, are used to provide a low channel resistance. 


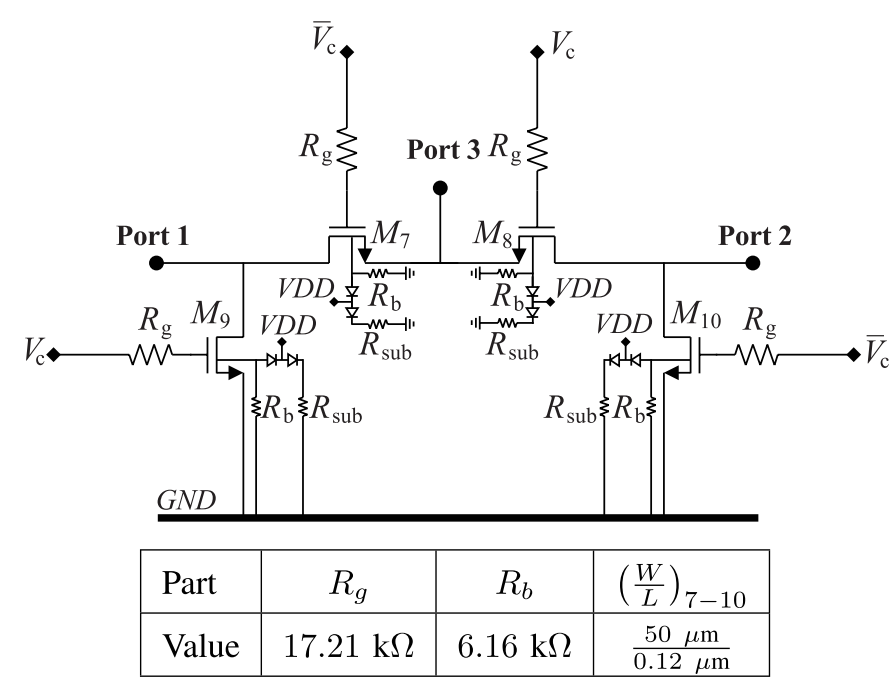

Fig. 6. Schematic of series-shunt SPDT switch.

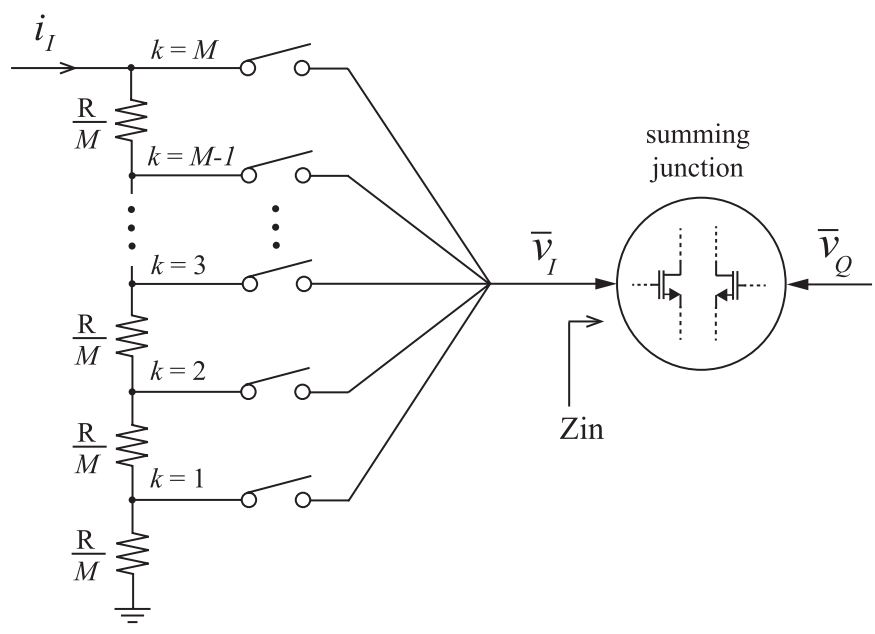

Fig. 7. High-level schematic to illustrate the operation of the fine vectorscaling step in the $I$ path (the $Q$-path passive scaling network is identical).

Increasing the size of $M_{7}$ and $M_{8}$, consequently, degrades the isolation of the switch in the OFF states. As shown in Fig. 6, two shunt transistors are utilized here to form a series-shunt SPDT switch and compensate for the isolation degradation, due to larger parasitic capacitors of series switches. Furthermore, deep n-well nMOS transistors, designed with body floating technique [16], are used to isolate the transistors' bodies from substrate and boost the switch performance in terms of lower insertion loss. The simulated results show that the insertion loss of the SPDT switch is $<1 \mathrm{~dB}$, and its isolation and return losses, in the whole frequency band, are negligible and better than 41 and $18 \mathrm{~dB}$, respectively, and it has negligible effect on the performance of the switches.

\section{Passive Vector-Scaling Circuit (Fine Scaling)}

Fig. 7 shows the concept of the second vector-scaling stage in the $I$ path (the $Q$-path scaling network is identical). A series resistor network is connected from the signal path to ground. A series switch is connected between each resistor node and the output path, and only one switch is turned ON at any given time. If there are $M$ identical resistors in the network and each has a value $R / M$, then the output voltage when the switch in the $k$ th branch is activated is

$$
\overline{v_{I}}=\left[\left(\frac{k R}{M}\right) / / Z_{\text {in }}\right] i_{I} \approx\left(\frac{k R}{M}\right) i_{I}
$$

and the approximation holds if $R \ll Z_{\text {in }}$, which is easily satisfied if $R$ is in the hundreds of ohms or a few kilohms, because the input terminals to the summing junction are nMOS gates. Recalling that $i_{I}=G_{m} v_{b, I}$, the overall scaling factor between the signals $\overline{v_{I}}$ and $v_{b, I}$ when the switch in branch $k$ is activated is

$$
A_{v}=k\left(\frac{R}{M}\right) G_{m} \mathrm{~V} / \mathrm{V}
$$

and the expressions for the maximum and minimum vectorscaling factors are

$$
\begin{aligned}
& A_{v, \text { max }}=R G_{\max } \quad k=M \\
& A_{v, \text { min }}=\left(\frac{R}{M}\right) G_{\text {min }} \quad k=1 .
\end{aligned}
$$

Using (2) and (5), the new bit resolution, $n^{\prime}$, of the phase shifter due to the fine-scaling resistive scaling network is

$$
\begin{aligned}
\left\lfloor n^{\prime}\right\rfloor & =\log _{2}\left[\frac{\pi}{\tan ^{-1}\left(\frac{(R / M) G_{\min }}{R G_{\max }}\right)}\right] \\
& \approx \log _{2}\left[\frac{\pi M}{G_{\min } / G_{\max }}\right]
\end{aligned}
$$

where the approximation $\tan ^{-1} x \approx x$ for small $x$ is invoked, because the fine scaling ensures that $A_{v \text {, min }} \ll A_{v \text {, max }}$. Expression (6b) enables the designer to determine how many resistors $(M)$ are needed in the second scaling step to obtain a target bit resolution once the $G_{m}$ tuning range of the OTAs has been established.

For the circuit under discussion, the $G_{m}$ tuning range chosen to minimize the rms phase and amplitude error of the phase shifter is $5.5-32 \mathrm{mS}$, which would yield a resolution of $4 \mathrm{~b}$. Since the target phase-shift resolution for this design is $6 \mathrm{~b}$, (6b) requires that $M=4$ to achieve the goal, and thus, the circuit used to carry out the fine-scaling step in this paper is shown in Fig. 8.

As noted in Section II, the phase response of $G_{m}$ will adversely impact the rms phase and amplitude error performance of the circuit and it is preferred to keep $V_{\text {tune within a }}$ region, where the $G_{m}$ phase variation is minimized. Therefore, during the experimental part of this paper, $V_{\text {tune }}$ for $k=2-4$ was limited to the range of $0.55-0.85 \mathrm{~V}$ to keep the phase variation of $G_{m}$ below $3^{\circ}$.

\section{E. Vector-Summation Circuit}

Fig. 9 shows the schematic of the summing circuit used here. $V_{b 1}, V_{b 2}$, and $V_{b 3}$ are the dc bias voltages. The $\bar{v}_{I}$ and $\bar{v}_{Q}$ signal vectors are incident at the gates of transistors $M_{12}$ and $M_{13}$, respectively. The drain currents of the cascode 


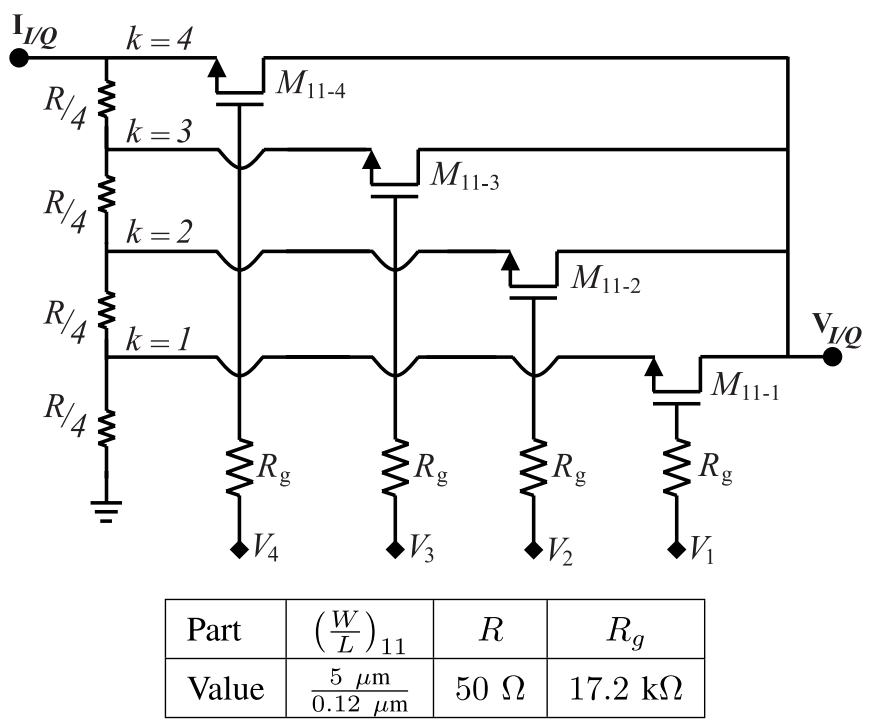

Fig. 8. Schematic of the four-resistor network used for fine vector scaling.

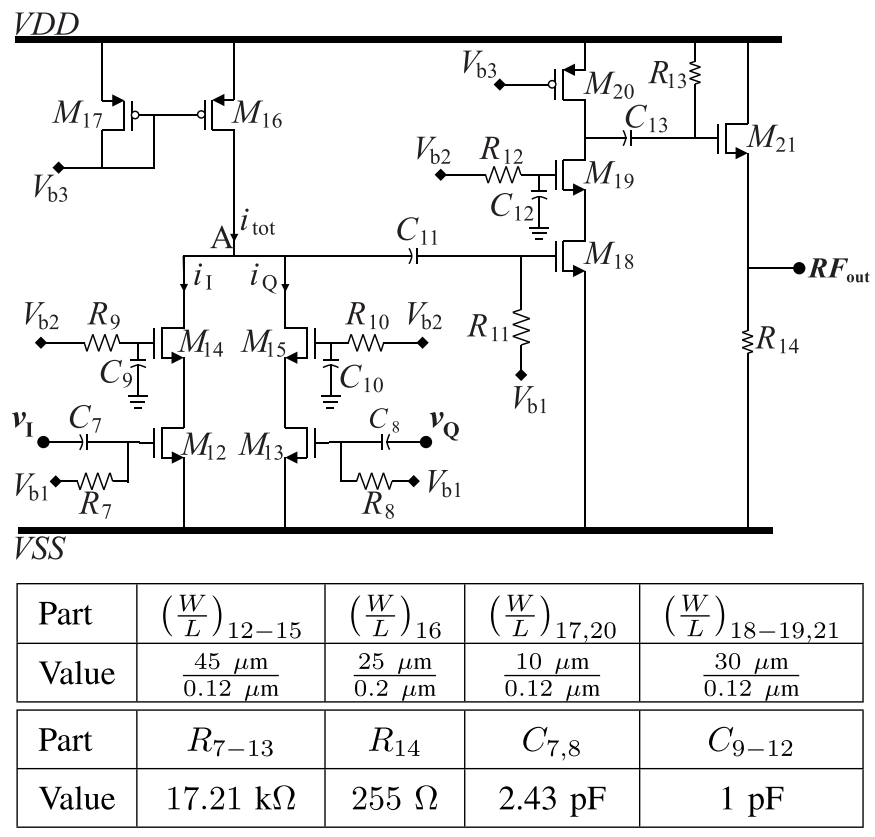

Fig. 9. Schematic of the vector-summation circuit.

transistors $M_{14}$ and $M_{15}$ are added at node A and are fed to a transimpedance amplifier. A source-follower $\left(M_{21}\right)$ is used as a buffer to isolate the phase shifter from the 50- $\Omega$ impedance environment of the measurement system.

\section{EXPERIMENTAL RESULTS}

The OTA-based active phase shifter is fabricated using the Global Foundries (formerly IBM) $0.13-\mu \mathrm{m}$ CMOS process. A microphotograph of the fabricated chip is shown in Fig. 10. The chip core occupies an area of $0.303 \mathrm{~mm}^{2}$ excluding bonding pads. The circuit draws a maximum of $28 \mathrm{~mW}$ of dc power from a $1.2 \mathrm{~V}$ supply.

An Agilent 8510C vector network analyzer was used for measuring the insertion phase and gain of the chip in different

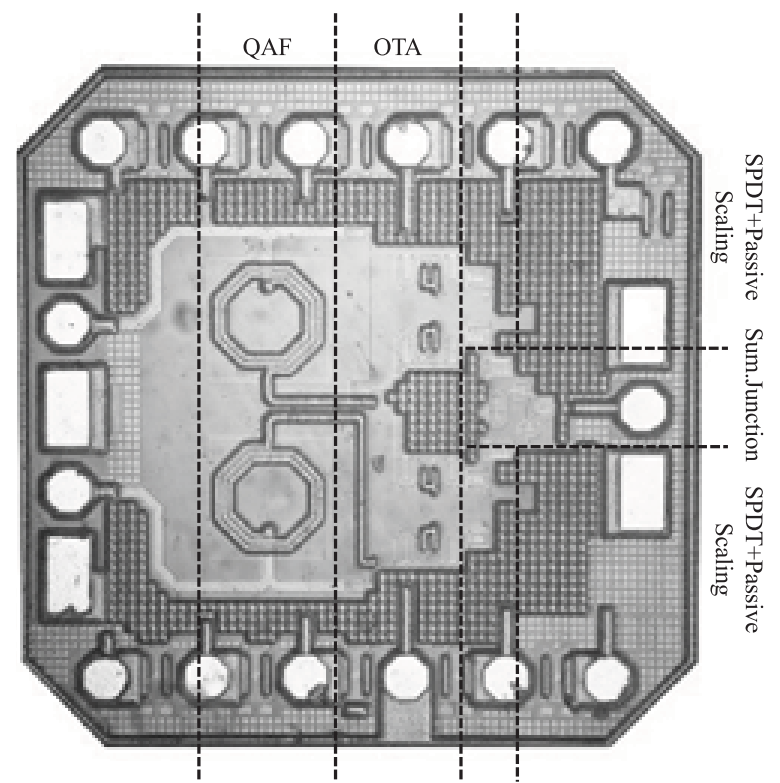

Fig. 10. Microphotograph of the fabricated active phase shifter.

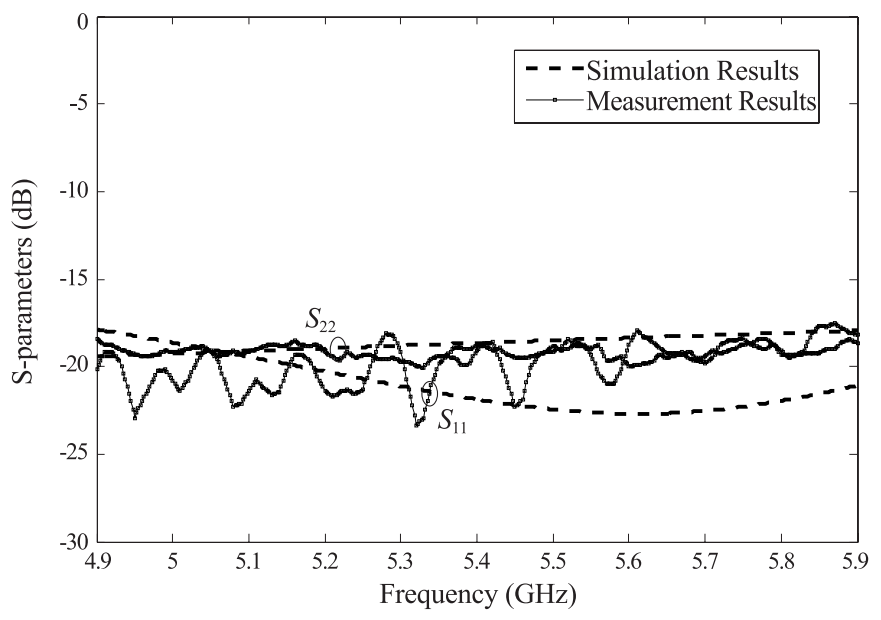

Fig. 11. Measurement and simulation results for input and output return losses.

phase settings. The chip's differential input signals are fed by an external hybrid coupler, Krytar 4010180 with around $0.5 \mathrm{~dB}$ loss in $5-\mathrm{GHz}$ band.

As noted earlier, the input and output matching circuitries of the phase shifter are independent of the phase shifter's phase setting. Therefore, the input and output reflection coefficients, $S_{11}$ and $S_{22}$, are provided for just one phase setting. Fig. 11 shows the input and output return losses. The measurement and simulation results agree for both $S_{11}$ and $S_{22}$. The return losses are better than $15 \mathrm{~dB}$ in the frequency band of interest. Required tuning voltages for $I$ and $Q$ paths, $V_{\text {tune, } I}$ and $V_{\text {tune, } Q}$, are varied with $10-\mathrm{mV}$ quantization steps, and their values and digital controls (refine bits) are provided in a lookup table.

Fig. 12 shows the unwrapped measured insertion phase response for 64 different phase states spaced $5.625^{\circ}$ apart (6-b resolution). Both the measured and ideal relative insertion 


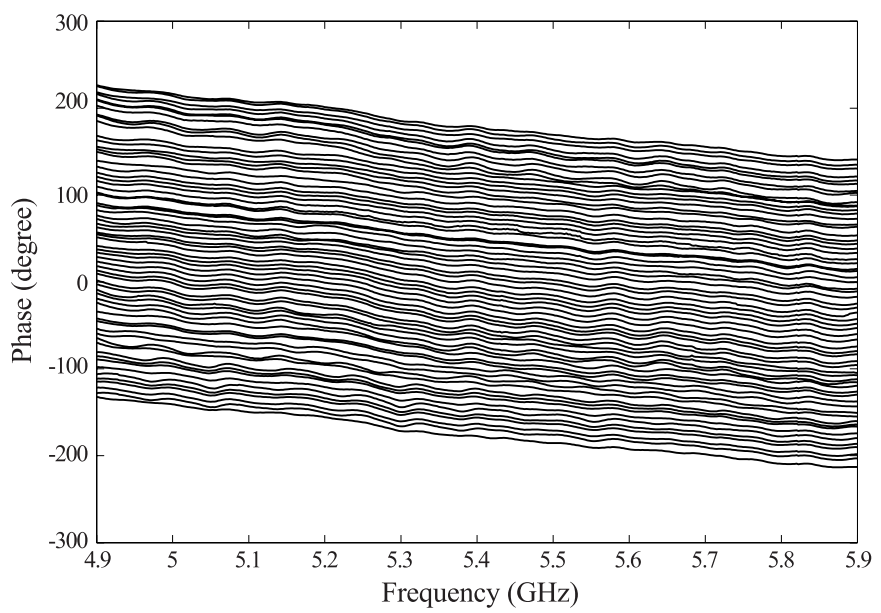

Fig. 12. Measured insertion phase for 64 output phases.

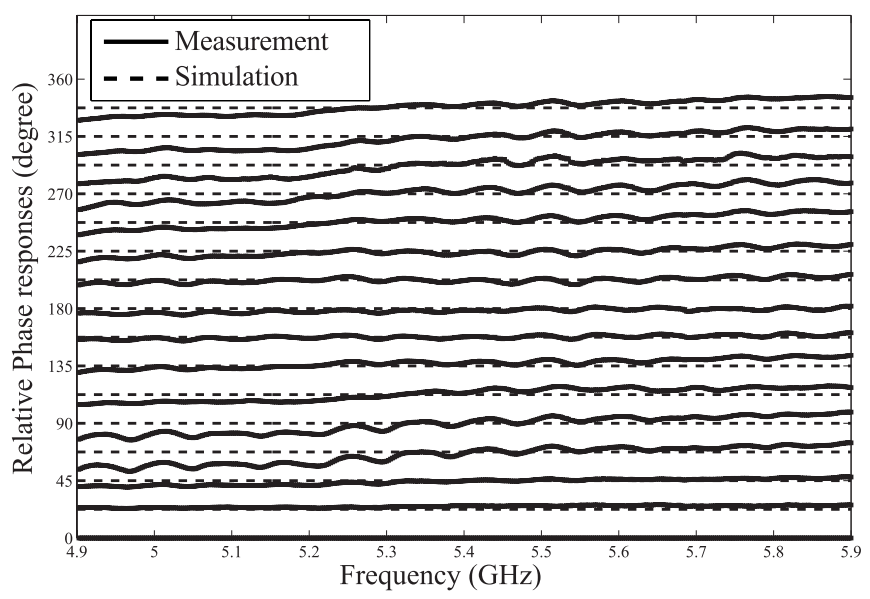

Fig. 13. Measured (solid lines) and ideal (dashed lines) relative insertion phase for 16 out of 64 possible states.

phases of the circuit are shown in Fig. 13. Only 16 out of the 64 possible curves are shown to avoid an overcrowded plot. As expected, the best agreement between the measured and ideal curves in Fig. 13 occurs at the design frequency of $5.4 \mathrm{GHz}$.

The rms phase error of a phase shifter can be calculated using (7), where $N$ is equal to number of phase states (64 in this paper) and $\theta_{\Delta i}$ is the difference between the phase angle produced by the phase shifter and the expected theoretical value. The rms phase error of the proposed phase shifter is $1.25^{\circ}$ at $5.4 \mathrm{GHz}$. The rms phase error as a function of frequency is shown in Fig. 14. The simulated and measured group delays are 0.11 and $0.14 \mathrm{~ns}$, respectively,

$$
\begin{aligned}
& \theta_{\Delta, \mathrm{RMS}}=\sqrt{\frac{1}{N-1} \times \sum_{i=2}^{N}\left|\theta_{\Delta i}\right|^{2}}\left({ }^{\circ}\right) \\
& A_{\Delta, \mathrm{RMS}}=\sqrt{\frac{1}{N} \times \sum_{i=1}^{N}\left|A_{\Delta i}\right|^{2}(\mathrm{~dB}) .}
\end{aligned}
$$

Fig. 15 shows the insertion gain responses for some of the phase states (16 states are chosen for better illustration). The rms gain error, calculated using (8) for all 64 settings, is always

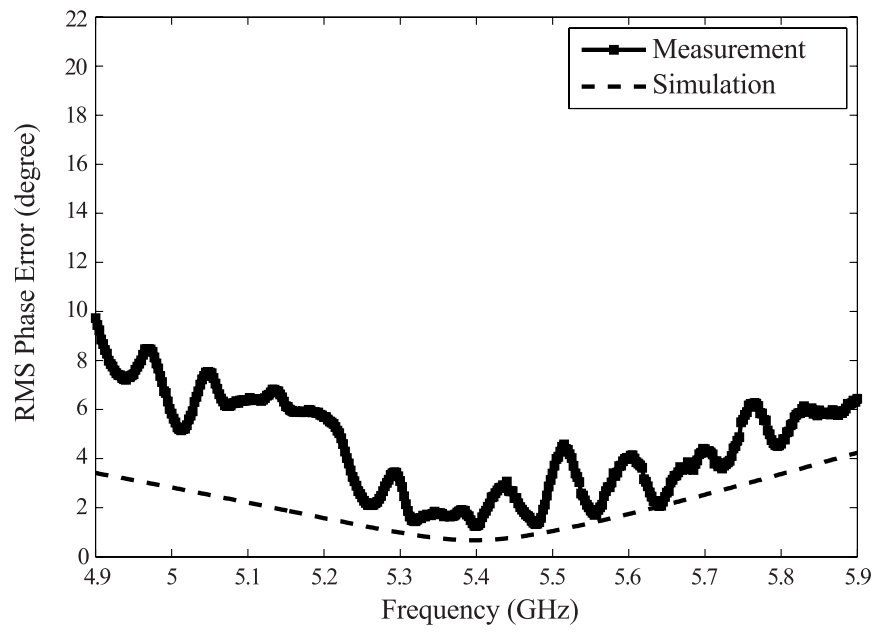

Fig. 14. RMS phase error.

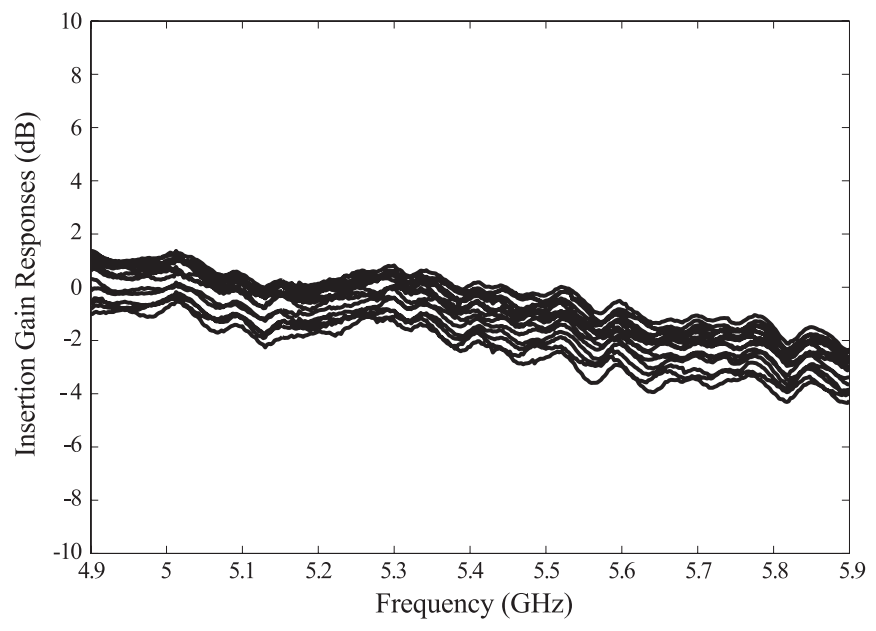

Fig. 15. Measured insertion gain for 16 states out of 64 phase settings (for more clear demonstration).

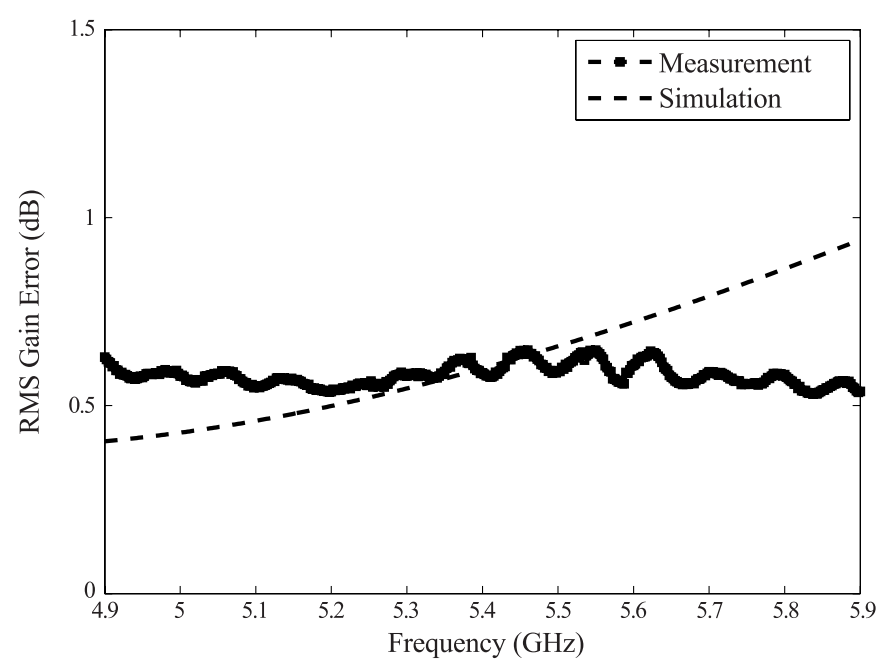

Fig. 16. RMS amplitude error.

less than $0.7 \mathrm{~dB}$, as shown in Fig. 16. The degradation of the measurement results from the simulations is $<0.5 \mathrm{~dB}$ and experiences its maximum as it reaches the frequency band's edges. Here, $\mathrm{A}_{\Delta i}$ is the deviation of the gain response in each 
TABLE I

Performance Summary and COMPARISON TABLE

\begin{tabular}{|c|c|c|c|c|c|c|c|}
\hline & units & This work & [6] & [7] & [8] & [9] & [10] \\
\hline Frequency band & $\mathrm{GHz}$ & $4.9-5.9$ & $6-18$ & $2-3$ & $2.3-4.8$ & $15-35$ & $0.5-6$ \\
\hline Phase range/resolution & degree / degree & $360 / 5.625$ & $360 / 22.5$ & $360 /-$ & $360 / 5.625$ & $360 / 22.5$ & $360 /-$ \\
\hline RMS phase error & degree & $1.25-9.7$ & $2.7-10$ & $1-5$ & $1.04-1.4$ & $4.2-13$ & $\leq 7$ \\
\hline RMS amplitude error & $\mathrm{dB}$ & $0.5-0.7$ & $0.5-1.7$ & $0.75-1.2$ & $0.2-1.1$ & $1-2.2$ & $\leq 0.95$ \\
\hline Insertion gain & $\mathrm{dB}$ & $-0.1 \pm 1.15^{*}$ & $-1.15 \pm 0.95$ & $1.5 \pm 1.5^{*}$ & $-1 \pm 1^{*}$ & $-4.25 \pm 9.25$ & $9 \pm 1$ \\
\hline Input $\mathrm{P}_{1 \mathrm{~dB}}$ & $\mathrm{dBm}$ & $-12.9 \pm 1$ & -5.4 & -13.5 & $1.8 \pm 1.2$ & -6.25 & $-19.5 \pm 2.5$ \\
\hline Supply voltage & $\mathrm{V}$ & 1.2 & 1.5 & 2 & 1.8 & 1.8 & 1.5 \\
\hline DC power & $\mathrm{mW}$ & 28 & 8.7 & 24 & 19 & 25.2 & 27.5 \\
\hline Chip area & $\mathrm{mm}^{2}$ & $0.303^{* *}$ & 0.45 & 0.38 & $0.652^{* *}$ & $0.19^{* *}$ & 1.21 \\
\hline$A_{\Delta, R M S} \times \theta_{\Delta, R M S}$ & $\mathrm{~dB}-{ }^{\circ}$ & $0.625-6.8$ & $1.35-17$ & $0.75-6$ & $0.21-1.54$ & $4.2-28.6$ & 6.65 \\
\hline
\end{tabular}

${ }^{*}$ at the mid-band frequency

** core size

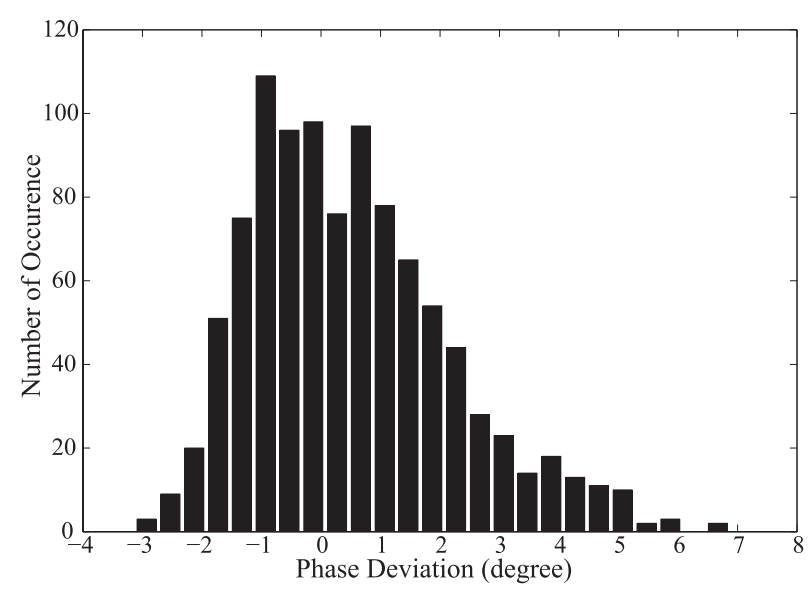

(a)

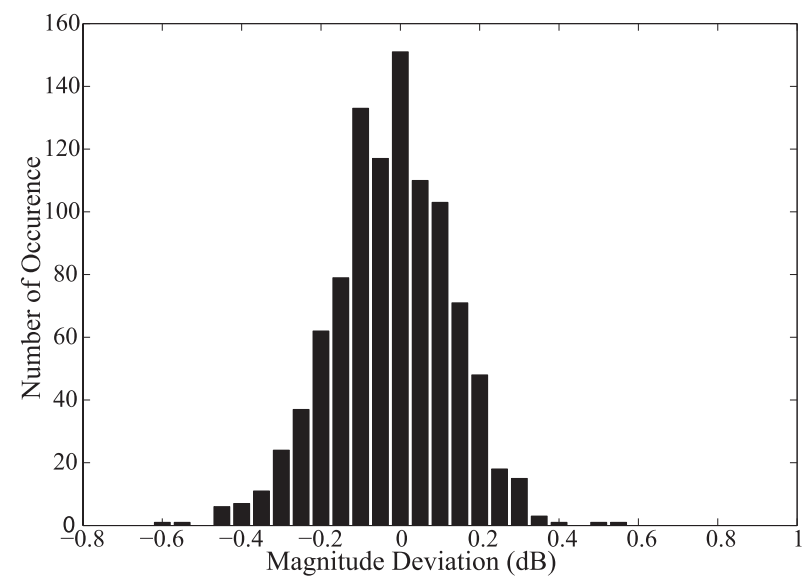

(b)

Fig. 17. Distribution of (a) phase and (b) magnitude deviation with mismatch in Monte Carlo simulation with 1000 runs.

phase state from the average insertion gain in decibel scales. The measured maximum peak to peak insertion gain error is -1.24 to $1.06 \mathrm{~dB}$ at $5.4 \mathrm{GHz}$, while its average value is $-1 \mathrm{~dB}$.

A Monte Carlo simulation was carried out to examine the effect of device mismatches on the amplitude and phase variations of the phase shifter. Fig. 17 shows the Monte Carlo results for a representative phase state of $0^{\circ}$. The standard deviation of the phase angle for eight phase states

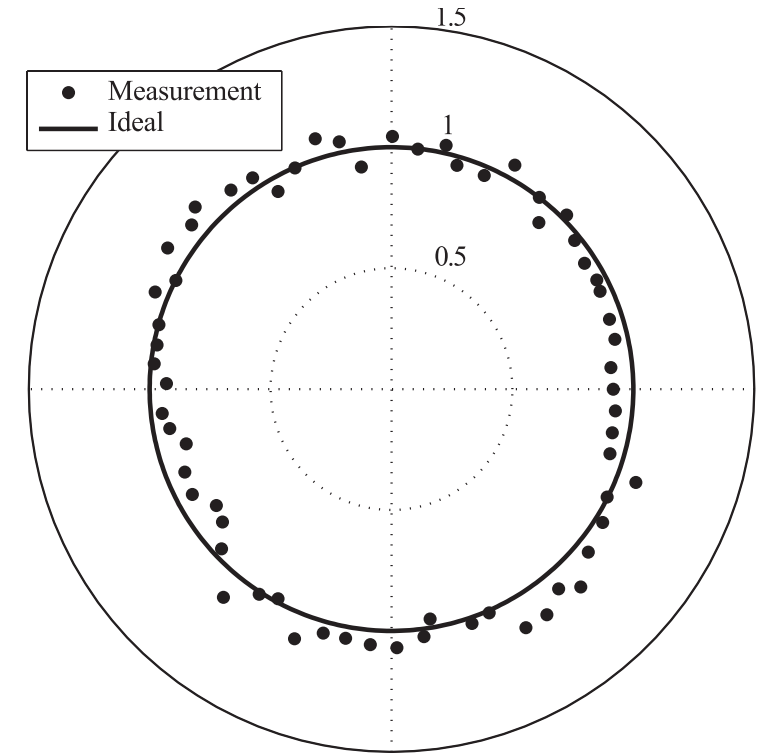

Fig. 18. Measured constellation diagram of the phase shifter.

around $360^{\circ}$ is always less than $1.7^{\circ}$, which is well below the resolution of the phase shifter $\left(5.625^{\circ}\right)$. Meanwhile, the standard deviation of the amplitude variation is below $0.15 \mathrm{~dB}$. The $72.5 \%$ of the occurrences (out of 1000 runs) were less than 0.25 of the least significant phase shift $\left( \pm 1.2^{\circ}\right)$ away from the mean value of that state. The polar plot, shown in Fig. 18, shows the uniform distribution for all 64 constellation states of the phase shifter, normalized to the average insertion gain.

Fig. 19 shows the measured output power versus input power to study the linearity performance of the phase shifter at $5.4 \mathrm{GHz}$. The input-referred $1-\mathrm{dB}$ compression point is $-12.9 \mathrm{~dB}$. The simulation results show that the $1-\mathrm{dB}$ compression point variation, for all the phase states, is less than $\pm 1 \mathrm{~dB}$. This is in accordance with the 2-dB insertion gain variation in different phase settings, reported by the measurements. The simulation result for the noise figure is also around $20 \mathrm{~dB}$ for all phase states.

A summary of the phase shifter's performance is outlined in Table I, in conjunction with a comparison with some of the previously reported designs in the similar CMOS technology. 


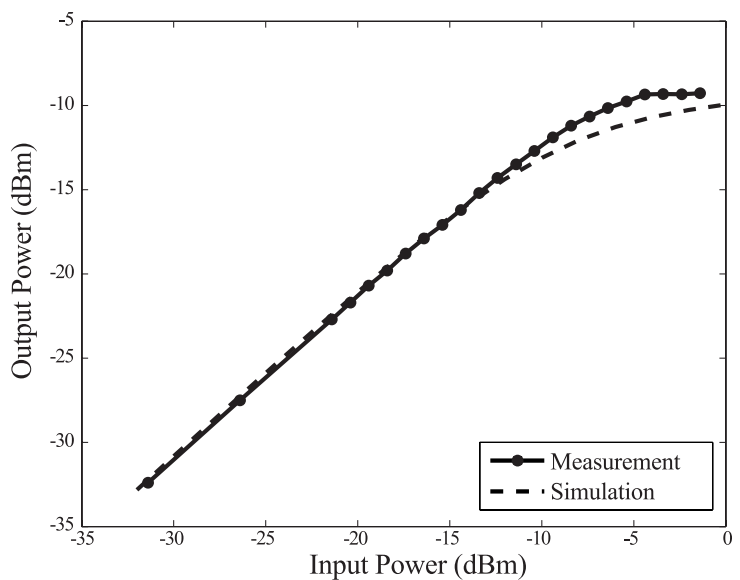

Fig. 19. Measured P1-dB compression point for the $0^{\circ}$ phase setting.

Table I also includes the calculation of a basic metric

$$
A_{\triangle, \mathrm{RMS}} \times \theta_{\Delta, \mathrm{RMS}}
$$

for each circuit consisting of the product of the rms amplitude and phase errors. The metric is calculated at the center frequency and has the units of $[\mathrm{dB}]\left[^{\circ}\right]$.

\section{CONCLUSION}

A high-accuracy low rms error phase shifter based on the vector-sum method is demonstrated in this paper. The use of coarse and fine scaling of the signal vectors is critical to reducing phase gaps between quadrants and for reducing therms phase and amplitude errors. Experimental tests on a fabricated prototype showed that therms phase error reached down to $1.25^{\circ}$, and therms amplitude error was $0.7 \mathrm{~dB}$ at the center frequency of $5.4 \mathrm{GHz}$, thereby validating the approach.

\section{ACKNOWLEDGMENT}

The authors would like to thank CMC Microsystems, Kingston, ON, Canada, for chip fabrication arrangements.

\section{REFERENCES}

[1] F. Ellinger, H. Jackel, and W. Bachtold, "Varactor-loaded transmissionline phase shifter at $C$-band using lumped elements," IEEE Trans. Microw. Theory Techn., vol. 51, no. 4, pp. 1135-1140, Apr. 2003.

[2] R. Amirkhanzadeh, H. Sjöland, J.-M. Redouté, D. Nobbe, and M. Faulkner, "High-resolution passive phase shifters for adaptive duplexing applications in sos process," IEEE Trans. Microw. Theory Techn., vol. 62, no. 8, pp. 1678-1685, Aug. 2014.

[3] O. D. Gurbuz and G. M. Rebeiz, "A 1.6-2.3-GHz RF MEMS reconfigurable quadrature coupler and its application to a $360^{\circ}$ reflectivetype phase shifter," IEEE Trans. Microw. Theory Techn., vol. 63, no. 2, pp. 414-421, Feb. 2015.

[4] M.-K. Cho, D. Baek, and J.-G. Kim, "An X-band 5 bit phase shifter with low insertion loss in $0.18-\mu \mathrm{m}$ SOI technology," IEEE Microw. Wireless Comp. Lett., vol. 22, no. 12, pp. 648-650, Dec. 2012.

[5] X. Tang and K. Mouthaan, "Design of large bandwidth phase shifters using common mode all-pass networks," IEEE Microw. Wireless Compon. Lett., vol. 22, no. 2, pp. 55-57, Feb. 2012.

[6] K.-J. Koh and G. M. Rebeiz, "0.13- $\mu \mathrm{m}$ CMOS phase shifters for X-, $\mathrm{Ku}$-, and K-band phased arrays," IEEE J. Solid-State Circuits, vol. 42, no. 11, pp. 2535-2546, Nov. 2007.

[7] Y. Zheng and C. E. Saavedra, "Full $360^{\circ}$ vector-sum phase-shifter for microwave system applications," IEEE Trans. Circuits Syst. I, Reg. Papers, vol. 57, no. 4, pp. 752-758, Apr. 2010.
[8] A. Asoodeh and M. Atarodi, "A full $360^{\circ}$ vector-sum phase shifter with very low RMS phase error over a wide bandwidth," IEEE Trans. Microw. Theory Techn., vol. 60, no. 6, pp. 1626-1634, Jun. 2012.

[9] S. P. Sah, X. Yu, and D. Heo, "Design and analysis of a wideband 15-35-GHz quadrature phase shifter with inductive loading," IEEE Trans. Microw. Theory Techn., vol. 61, no. 8, pp. 3024-3033, Aug. 2013.

[10] H. Zijie and K. Mouthaan, "A $0.5-6 \mathrm{GHz} 360^{\circ}$ vector-sum phase shifter in 0.13- $\mu \mathrm{m}$ CMOS," in IEEE MTT-S Int. Microw. Symp. Dig., Jun. 2014, pp. 1-3.

[11] Y. Kim, S. Kim, I. Lee, M. Urteaga, and S. Jeon, "A 220-320-GHz vector-sum phase shifter using single Gilbert-cell structure with lossy output matching," IEEE Trans. Microw. Theory Techn., vol. 63, no. 1, pp. 256-265, Jan. 2015.

[12] C. Quan, S. Heo, M. Urteaga, and M. Kim, "A $275 \mathrm{GHz}$ active vectorsum phase shifter," IEEE Microw. Wireless Compon. Lett., vol. 25, no. 2, pp. 127-129, Feb. 2015.

[13] J. Xu, C. E. Saavedra, and G. Chen, "Wideband microwave OTA with tunable transconductance using feedforward regulation and an active inductor load," in Proc. 8th IEEE NEWCAS Conf., Jun. 2010, pp. 93-96.

[14] Y. Zheng and C. E. Saavedra, "A microwave OTA using a feedforwardregulated cascode topology," in Proc. IEEE ISCAS, May 2007, pp. $1887-1890$

[15] Y. Zheng and C. E. Saavedra, "Feedforward-regulated cascode OTA for gigahertz applications," IEEE Trans. Circuits Syst. I, Reg. Papers, vol. 55 , no. 11 , pp. 3373-3382, Dec. 2008.

[16] B.-W. Min and G. M. Rebeiz, " $K a$-band low-loss and high-isolation switch design in 0.13- $\mu \mathrm{m}$ CMOS," IEEE Trans. Microw. Theory Techn. vol. 56, no. 6, pp. 1364-1371, Jun. 2008.

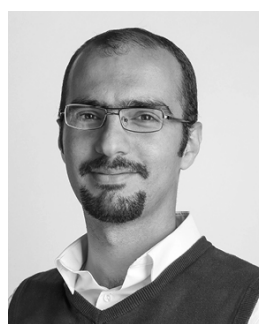

Mohammad-Mahdi Mohsenpour (M'14) received the B.Sc. degree in electrical engineering from the University of Mazandaran, Babol, Iran, in 2009, the M.Sc. degree in communications engineering, fields and waves, from the University of Tehran, Tehran, Iran, in 2012, and is currently pursuing the Ph.D. degree at the Gigahertz Integrated Circuits Group, Queen's University, Kingston, ON, Canada.

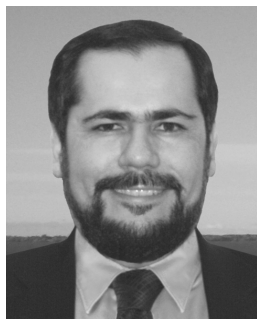

Carlos E. Saavedra (S'92-M'98-SM'05) received the B.Sc. degree in electrical engineering from the University of Virginia, Charlottesville, VA, USA, in 1993, and the Ph.D. degree in electrical engineering from Cornell University, Ithaca, NY, USA, in 1998.

$\mathrm{He}$ was a Senior Engineer with the Millitech Corporation from 1998 to 2000. In 2000, he joined Queen's University, Kingston, ON, Canada, where is currently a Professor. He served as the Graduate Chair of the Department of Electrical and Computer Engineering from 2007 to 2010.

Prof. Saavedra is a three-time recipient of the third-year ECE Undergraduate Teaching Award at Queen's University. He is an Associate Editor of the IEEE TRANSACTIONS ON MICROWAVE THEORY AND TECHNIQUES, a Member of the TPRC of the IEEE International Microwave Symposium (IMS), and a Member of the IEEE NEWCAS Conference Steering Committee. He was the Section Chair of the Natural Sciences and Engineering Research Council of Canada Discovery Grants Evaluation Group 1510 and Chair of the IEEE MTT-S Technical Coordinating Committee 22 on Signal Generation and Frequency Conversion. He served on the Steering and Technical Program Committees (TPCs) of the 2012 IEEE MTT-S IMS and was a Member of the IEEE RFIC Symposium TPC from 2008 to 2011 . 